\title{
The $\mu$-flown: a novel device for measuring acoustic flows
}

\author{
Hans-Elias de Bree *, Peter Leussink, Twan Korthorst, Henri Jansen, Theo S. J. Lammerink, \\ Miko Elwenspoek \\ MESA Research Institute, University of'Twente, PO Box 217,7500 AE Ensthede. Netheriunds
}

\begin{abstract}
An acoustic wave consists of two elements, the acoustic pressure and the acouslic flow. Up to now one has to measure the pressure and calculale the flow to determine the acoustic flow, so it would be convenient to have a sensor that is able to measure acoustic flows. At the University of Twente a novel device has been de veloped which fullils this need. In this paper a short introduction to the governing principles of this dynamic flow sensor, the fabrication process, the electronics and some of its interesting applications will be presented. This micromachined device measuring acoustic flows is called the microflown or $\mu$-fiown.
\end{abstract}

Keywords: Acoustic flow ; Microllown

\section{The acoustic flow seasor}

The starting poinl of the investigation is the micro liquid flow sensor [1]. This sensor consists of three resistors located in the middle of a channel (Fig. 1). The two outermost resistors are used as sensors, while the resistor located in the middle is used as a heater. The dimensions of the sensor are in hundreds of micrometres. An applied flow causes a difference in temperature between the upstrean and downstream sensors. The change of resistance in both resistors can be measured and leads to an electrical output proportional to the applied flow. While the displacement of the heated gas is measured, the sensitivity is inversely proportional to the frequency. Furthermore, the thermal time constant of the sensing elements will cause a sensitivity inversely proportional to the frequency. For low frequencies the dynamic model will converge to the (quasi-)static flow-sensor model. In contrast with the model of the static flow sensor [1], in which the gas flow transports the generated heat out of the system, there is no net heat transport in the mode! of the dynamic flow sensor.

\footnotetext{
* Corresponding author. Phone: + 31534894438 . Fax: + 31534309547 E-mail: H.E.deBree@el.utwente.nl.
}

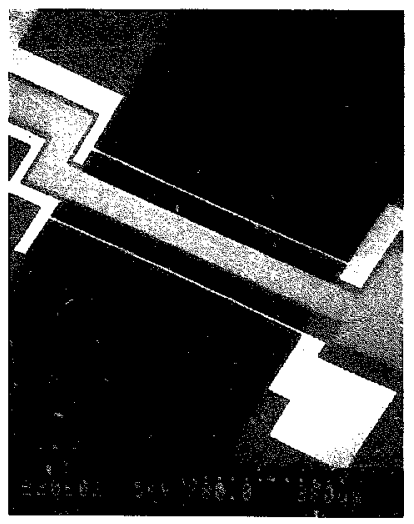

Fig. 1. Thic $\mu$-flown. The spacing between the $\mathrm{Si}_{x} \mathrm{~N}_{y}$ bridges is $30 \mu \mathrm{m}$. The width of the sensors is $5 \mu \mathrm{m}$ and the heater is $100 \mu \mathrm{m}$ wide.

\section{Acoustic theory}

The acoustic domain can be described with two physical quantities, pressure and volume flow. However, in the field of acoustics the particle velocity is used instead of the volume 
flow. The particle velocity is defined as the volume flow divided by the surface in which the wave propagates. The ratio of the pressure and the particle velocity is the specific acoustic impedance [2]. The specific acoustic impedance for a number of typical cases will be discussed below. [2]:

The specific acoustic impedance of open air is given by

$Z_{s}(r)=\rho c \frac{\mathrm{j} r(\omega / c)}{1+\mathrm{j} r(\omega / c)}$

using $\rho$ as the density of the medium, $c$ the velocity of sound in the medium and $r$ representing the distance to the acoustic source. For a frequency of $100 \mathrm{~Hz}$ the ratio $\mathrm{c} / \omega$ is $55 \mathrm{~cm}$. For a larger radius Eq. (1) is simplified to $Z_{\mathrm{s}}=\rho c$.

Another case is a short tube with an acoustic source on one side and on the other a stiff, reflecting wall. The acoustic impedance in the tube in the longitudinal axis is dependent on both frequency and position [2].

The third environment is a tube of an infinite length. In practice the length will be finite, but it can still be used if the measurement takes place before the first reftection reaches the measuring point. The specific acoustic inpedance of an infinite tube is [3]

$Z_{3}=\rho c$

In order to calibrate the $\mu$-flown, a micromachined device for measuring acoustic flows, it has to be remembered that this is a new device, so a reference $\mu$-flown is not available. The solution of this problem has been found in using a reference pressure microphone and $\mathrm{Eq}$. (2). This impedance is independent of position and frequency, which makes it well suited for transfer-function measurements.

The last definition we would like to present here is the intensity. Intensity equals the product of the pressure and the particle velocity. Therefore it is a vector and related to the propagation of the power incorporated in an acoustic wave.

\section{Boundary conditions for plane-wave propagation in a cylindrical tube}

For frequencies lower than $f_{s}$, called the cut-off frequency. c.. Iy plane waves exist and non-plana waves vanish exponentially $[4]$. The cut-off frequency is given by

$f_{c}=\frac{c}{d 1.71}$

where $d$ is the dianeter of the tube.

Other types of waves are generated when the tube starts to resonate in the radial-circum ferential flexural mode [5]. This effect is neglected here. In order to make a plane-wave approximation, the viscous boundary layers should be thin [4]: $f_{\min } \gg \frac{2 \nu}{\pi d^{f}}$

where $\nu$ represents the kinematic viscosity of air.

Because of the finite length of a tube and the reflections at the end, the lowest frequency that can be measured is

$\hat{f}_{\min } \approx \frac{c}{2 l}$

where $l$ is the length of the tube.

\section{Measuriag electronics: the 'Wheatstone Gadget'}

The Gadget is a new type of circuit especially designed to measure the small differential resistor variations of the $\mu$ flown (Fig. 2).

The transfer function of the Gadget is [6]

$\Delta V_{\mathrm{our}}=2 I R_{2} \frac{\Delta R_{\mathrm{diri}}}{R}$

in which $\Delta V_{\text {out }}$ is the a.c. variation in the output voltage as a result of $\Delta R_{\text {diff }}$, the differential resistance variation of $R$, the initial value of the $\mu$-flown resistor. The d.c. bias current $I$ is defined as

$I=\frac{E-U_{\mathrm{BE}}}{R_{1}+R}$

in which $E$ is the power-supply voltage and $U_{\mathrm{BE}}$ the baseemitter voltage ( $\left.U_{\mathrm{BE}} \approx 0.6-0.7 \mathrm{~V}\right)$. The d.c. bias condition of the Gadget is

$V_{\text {out }}=E-I R_{2}>U_{\mathrm{E} . \mathrm{T} 2}+U_{\mathrm{CE} \text { min }} \approx U_{\mathrm{B}}$

Substituting Eq. (7) in Eq. (8), the d.c. bias condition yields

$R_{1} \geq R_{2}$

The d.c. output voltage is

$V_{\text {unt,d.c. }}=E-I R_{2}$

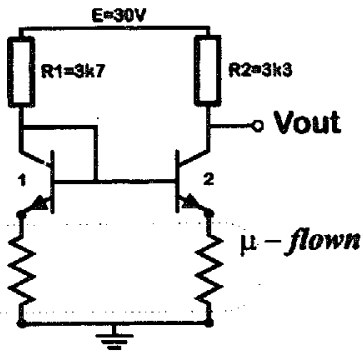

Fig. 2. Measuring electronics: the Gadget. 
The signal-to-noise ratio of the Gadget is [6]

$$
\left(\frac{\mathrm{S}}{\mathrm{N}}\right)_{\mathrm{Gadzct}}=\frac{I \sqrt{R} \frac{\Delta R}{R}}{\left\{q \cdot \mathrm{BW}\left[2 U_{\mathrm{T}}\left(\frac{T_{\mathrm{s}}}{T}+\frac{r_{\mathrm{bb}}}{R}\right)+\frac{U_{\mathrm{T}}^{2}}{I R}+\frac{U_{\mathrm{T}}}{2 \alpha_{\mathrm{FE}}}\left(\frac{r_{\mathrm{bb}}}{R}+2\right)+\frac{I R}{\alpha_{\mathrm{FE}}}\left(1+\left(1+\frac{r_{\mathrm{bb}}}{R}\right)\right)\right]\right\}^{2}}
$$

in which $q$ is the charge of an electron, BW the bandwidth of the signal, $U_{\mathrm{T}}$ the thermal voltage $(25 \mathrm{mV}), T_{\mathrm{s}}$ the sensor temperature, $T$ ambient temperature, $r_{\mathrm{bb}}$ the base series resistance of the used bipolar junction trasistor (BJT) and $\alpha_{\mathrm{FE}}$ the d.c. forward current gain.

The Gadget Duo Sensation (Fis, 3) consists of two Gadgets. It is capable of measuring the sum signal of two $\mu$-fowns at the same time and can be ised with either voltage or current output.

Using this Gadget in the current-output mode, the input of the next electronics has to be of low impedance and have a voltage of $0.5 E$. The output current is

$\Delta I_{\text {out }}=2 I\left(\left[\frac{\Delta R}{R}\right]_{2}-\left[\frac{\Delta R}{R}\right]_{1}\right)$

The bias current is given by

$I=\frac{E-2 U_{\mathrm{BE}}}{2 R+R_{\mathrm{C}}}$

For voltage output the following electronics has to be of high input impedance. The PNP-Gadget is an active load [7] for the NPN-Gadget and vice versa. This way it is possible to get a large transfer function with a relatively low powersupply voltage. If $U_{\mathrm{ou}} \approx E / 2$, the transfer function is

$$
\begin{aligned}
\Delta U_{\text {out }} & =\Delta I_{\text {oul }}\left(r_{\mathrm{a}, \mathrm{PNP}}|| r_{\mathrm{D}, \mathrm{NPN}}\right) \\
& =\frac{U_{\mathrm{A}, \mathrm{NPN}} U_{\mathrm{A}, \mathrm{PNP}}}{U_{\mathrm{A}, \mathrm{NPN}}+U_{\mathrm{A}, \mathrm{PNP}}} \frac{\Delta I_{\text {oul }}}{I}
\end{aligned}
$$

using $U_{\mathrm{A}}$ as the Early voltage and $r_{0}$ is the output voltage of a BJT. Note that this transfer function is independent of the

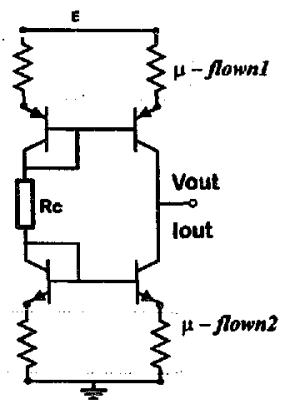

Fig. 3. The Gadget Duo Sensation. bias current. The signal-to-noise ratio is approximately the same as Eq. (11).

\section{The fabrication process}

The method that we are using to manufacture the $\mu$-flown is a derivative of the process for the flow sensor as described in Ref.[1]. The process is relatively simple and straightforward. We use low-pressure chemical-vapour deposition techniques to dispusit the layers and reactive ion etching to perform dry etching. We start with a silicon wafer of $380 \mu \mathrm{m}$ thickness. First we deposit a polysilicon layer, which will be used as a sacrificial layer. After patterning by photolithography and dry etching, we deposit a silicon nitride layer. This iayer is also patterned by photolithography and dry etched. The next step is carried out by deposition of the metal layer by sputtering. The metals that we use are chromium and gold. The pattern in the metal layer is not created by etching but with lift-off. The last step in the process is the etching of the channel. The silicon wafer is already patterned with the silicon nitride layer and we use a $\mathrm{KOH}$ solution to etch an approximately $300 \mu \mathrm{m}$ deep channel. This wet-etching method is anisotropic and gives defined channel walls, but a time stop is needed to define the depth of the channel. After the wet etching the samples have to be sawed, mounted and bonded.

\section{Measurements}

Measurements of the described acoustic phenomena have been performed with the $\mu$-flown in combination with a conventional pressure microphone. The experimental set-up consists of a $20 \mathrm{~m}$ long tube in which the $\mu$-flown and a reference microphone are mounted, see Fig. 4. By generating a burst and measuring within a specific time span, the influence of reflections can be avoided.

In an acoustic wave a pressure wave and a flow wave are inextricably related. Figs. 5 and 6 show how the pressure and flow are altered after reflection. The first bursts are generated

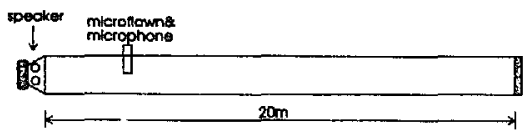

Fig. 4. Measurement set-up I. 


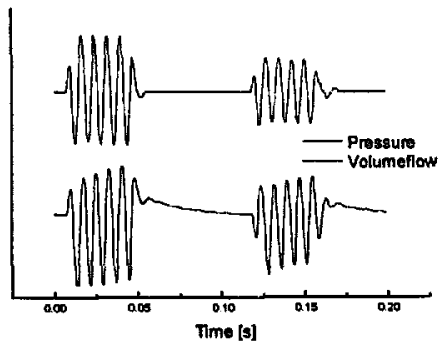

Fig. 5. Acoustic response of a rigidly closed ube: phase shif after reflection in particle flow and no phase shift in pressure.

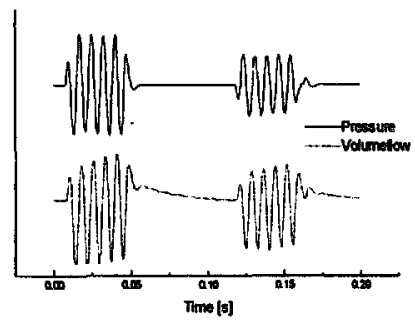

Fig. 6. Acoustic response of an open tube: phase shift after reflection in pressure and no phase shift in particle flow.

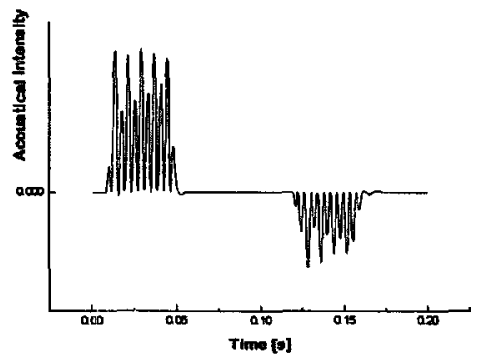

Fig. 7. Sound intensity of a forward and backward propagating wave in a rigidly closed tube.

by forward propagating waves while the second bursts are generated by backward propagating waves.

Another good example of what is possible with the combination of microphone and $\mu$-flown is shown in Fig. 7: while the intensity of the forward propagating wave is positive, the intensity of the backward propagating wave is negative.

The sensitivity, i.e., the ratio of the output voltage and the applied particle velocity, of the $\mu$-flown is shown in Fig. 8 . The acoustic impedance is $\rho c \approx 448 \mathrm{~kg} \mathrm{~m}^{-2} \mathrm{~s}^{-1}$ thus a sound pressure level of $60 \mathrm{~dB}$, which equals $0.02 \mathrm{~Pa}$, will result in a particle velocity of $45 \mu \mathrm{m} \mathrm{s}^{-1}$.

The output of a set of two $\mu$-flowns in combination with a Gadget Duo Sensation (Fig. 3) as a function of the acoustic pressure applied was measured by a frequency of $100 \mathrm{~Hz}$ (Fig. 9). The power-supply voltage, $E$, was $40 \mathrm{~V}$ and both sensor and heater currents were $10 \mathrm{~mA}$. An output of $2 \mathrm{mV}$, which equals a sound pressure level of $50 \mathrm{~dB}$, could be measured.

A different set-up is used in the next measurement: $a 40 \mathrm{~m}$ long tube with a loudspeaker at each end (Fig. 10).

One speaker generates a $150 \mathrm{~Hz}$ burst and the other a 200 $\mathrm{Hz}$ burst. The $\mu$-flown and microphone are situated in the middle, measuring both acoustic waves travelling in opposite directions (upper two curves, Fig. 11).

Because in forward and backward travelling waves the particle flow is $180^{\circ}$ phase shifted, the sum signal of the upper

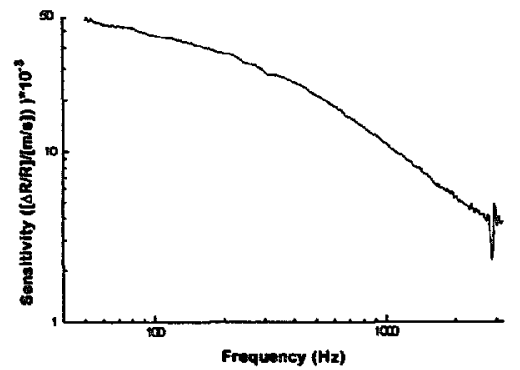

Fig. 8. Sensitivity of the $\mu$-ilown. The shs configuration was used. The sensor and heater current were each $10 \mathrm{~mA}$.

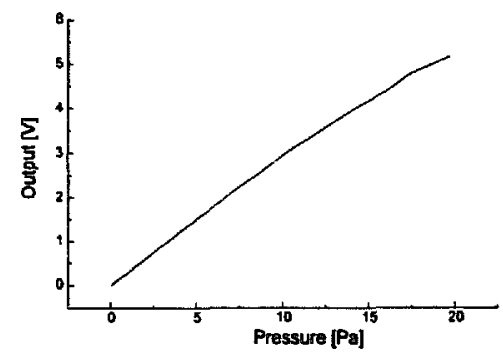

Fig. 9. The oulput of two $\mu$-flowas in combination of a Gadget Duo Sensation as a function of the acoustic pressure for a frequency of $100 \mathrm{~Hz}$.

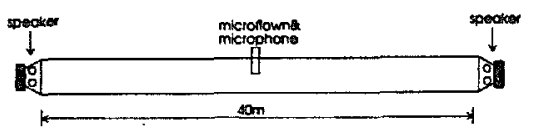

Fig. 10. Measurement set-up II. 


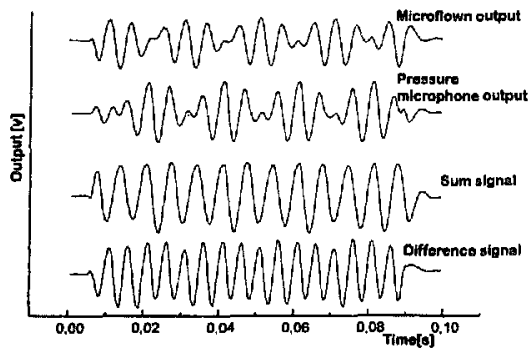

Fig. I 1. Combining a pressure inicrophone with a $\mu$-flown, one can create a unipolar microphone.

two curves results in the forward travelling wave. The backward travelling wave is obtained by subtracting the output of the $\mu$-flown from the outpul of the pressure microphone.

\section{Multiple configurations}

In the applied set-up multiple configurations of the dynamic flow sensor are possible. The above-mentioned configuration is called a sensor heater sensor (shs) type of $\mu$ flown, describing the geometrical structure. Other possible configurations are sensor sensor (ss) and sensor heater (sh); see Fig. 12.

It is possible to create a microflown with only two sensors, both heated by a current. This type of $\mu$-flown has two fundamental advantages: the structure is simplified and measurements with a larger current gives a larger sensitivity to the applied acoustic flow. Furthermore, only one sensor with a heater appears to perform. It is basically a hot-wire anemometer [8] but enhanced with a heater ncarby. The interesting features of this type of $\mu$-flown are the fact that the output increases with the applied heater current and the possibility, in contrast to the hot-wire anemometer, of detecting the direction of the How (Fig. 13). The characteristic double frequency of a hot-wire anemometer vanishes with the increasing heater current.

A remark about the configurations: it is possible to enlarge the sensor current. This causes the temperature of the sensor to rise and an increase of the sensitivity $(\Delta R / R)$ of the various types of $\mu$-flown. A sensor is called a hot sensor when its heat production is not negligible compared with that of the heater. In order to compare the various $\mu$-flown types, the sensitivity is a convenient value and is defined as the output of the $\mu$-flown ( $\Delta R / R$ in promille) at a frequency of $100 \mathrm{~Hz}$,

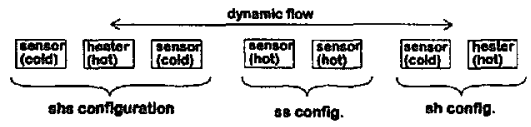

Fig. 12. Various microflown configurations.

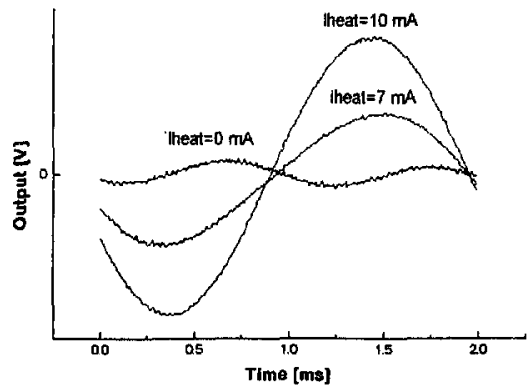

Fig. 13. Response of the combination of the $\mu$-flown and mensuring elec. tronics as a function of the heater current in a heater sensor configuration. The sensor current was $10 \mathrm{~mA}$.

Table 1

Sensilivity measurements of the various types of $\mu$-flowns

\begin{tabular}{llll}
\hline Sensor current & shs & ss & sh \\
\hline $2.5 \mathrm{~mA}$ & $0.17 \% \mathrm{~m}$ & N.A. & $0.05 \% \circ$ \\
$10 \mathrm{~mm}$ & $0.3 \% \circ$ & $0.17 \%$ & $0.08 \% \circ$ \\
\hline
\end{tabular}

a sound pressure of $100 \mathrm{~dB}(2 \mathrm{~Pa})$ and measured with a specific acoustic impedance $\rho c=448 \mathrm{~kg} \mathrm{~m}^{-2} \mathrm{~s}^{-1}$. Sensitivity measurements are presented in Table 1.

\section{Applications}

The combination of a dynamic flow sensor and a pressure sensor opens up a range of possible applications. With this set-up it is possible to perform an analysis of the direction of energy in both gases and liquids (Fig. 7). By subtracting and adding the adjusted signals of a $\boldsymbol{\mu}$-flown and pressure microphone, one can derive a stereo signal measured at one point. Another application is the measurement of the impedance in a system filled with liquid or gas, which gives information about the density and viscosity. Finally, a completely different application may be the use of a $\mu$-flown as a feedback sensor in a loudspeaker system.

\section{Conclusions}

We have developed a small and casy-to-operate dynamic flow sensor, the $\mu$-flown. When using it, one has to take into account the $1 / f$ behaviour. A significant improvement of the sensitivity of the complete device is to be expected from the combination of the $\mu$-flown and the Wheatstone Gadget, when used in other configurations. The $\mu$-flown makes it possible to measure the particle velocity of an acoustic wave. 
In combination with a microphone, it is possible to determine the direction of propagation of an acoustic wave.

\section{Further research}

Our priority is to develop a more detailed analytical model, and to gain more insight into the temperature distribution. Finite-element simulations will also be done.

With this insight it should be possible to design a more optimal $\mu$-flown with regard to the transfer function (increasing $\Delta R / R$ ). So far we have only investigated the constantpower principle; next we are going to investigate the constant-temperalure principle.

\section{Acknowledgements}

Pedro Roodenburg, who did the first promising experiments; Arnoud van der Wel for analysing the Wheatstone Gadget in detail and Edwin Potman and Jos Knippen for their technical assistance are grate fully acknowledged.

\section{References}

[1] T.S.J. Lammerink, N.R. Tas, M. Elwenspoek and J.H. J. Fluitman, Micro liquid flow sensor, Sensers and Acruators A, 37-38 (1993) 45-50.

(2) L. Beranek, Acoustics, McGraw-Hill, New York, 1954, pp. 16-40.

[3] P.J. Leussink and M.T. Korthorst, Inleiding Flierefluiten, MESA intermal report. University of Twente. 1994.

[4] A. Hirschberg and S.W. Rienstra, An introduction to acoustics, IWDE report 92-06. version 1/6/92, February 1992.

[5] R.D. Blevins, Formulas far Natural Frequency and Mole Shape. Kreiger, Malabar, FL, 1995.

(6) H-E. de Bree, P.J. Leussink, M.T. Konhorst, Y. Bäcklund and H.V. Jansen. The Wheastone gadget; a simple circuit for measuring differential resistance variations, MME '95. Copentagen. Denuterk. Sept. 1995, pp. 201-204.
[7] P.J. Gray and R.G. Meyer. Anelysis und Desigrt of Analogue Integrated Circuits, Wiley, New Yoik, 1993, pp. 287.

[8] S. Baker. An acoustic intensity meter, J. Acoust. Soc. Am., 27 (1955) 269-273.

\section{Biographies}

$H$. $E$. de Bree studied electrical engineering at the University of Twente in Enschedc. In 1994 he graduated from the Integrated Circuits and Electronics group. Ai the moment he is working for his Ph.D. at the Transducers and Materials Science group. In February 1994 he invented the $\mu$-flown.

P.J. Leussink started his studies of electrical engineering at the University of Twente in 1989 . He will receive his M.Sc. from the Transducers and Materials Science group in 1996.

M.T. Korthorsf started studying electrical engineering at the University of Twente in 1990 . He is now a graduate in the Transducers and Materials Science group.

H.V.Jansen studied eicctrical engineering at the University of Twente in Enschede from 1986 to 1991. In 1992-1996 he worked on his Ph.D. in the field of surface micromachining and developed the Black Silicon Method. He received his Ph.D. in April 1996. He is now working as technological assistant at the MESA Research Institute.

Theo Lammerink studied electrical engineering at the University of Twente in Enschede. In 1983 he joined the Transducers and Materials Science group as assistant professor. He received his $\mathrm{Ph} . \mathrm{D}$. in 1990 in the field of optical and micromechanical transducers and transducer systems.

$M$. Elwenspoek graduated from Freie Universität Bertin in 1977 on the physics of liquids. He received his Ph.D. in 1983 on nuclear quadrupolar relaxation in liquid alloys at the Freie Universität Berlin. Since 1987 he has been head of the Micromechanics Department at the University of Twente. 